\title{
The DNA Replication Stress Hypothesis of Alzheimer's Disease
}

\author{
Yuri B. Yurov, ${ }^{1,2,3}$ Svetlana G. Vorsanova, ${ }^{1,2,3}$ and Ivan Y. Iourov ${ }^{1,2}$ \\ ${ }^{1}$ Laboratory of Cytogenetics and Genomics of Psychiatric Disorders, Mental Health Research \\ Center, Russian Academy of Medical Sciences, Moscow 119152, Russia \\ ${ }^{2}$ Laboratory of Molecular Cytogenetics of Neuropsychiatric Diseases, Institute of Pediatrics \\ and Children Surgery, Minzdravsotsrazvitia, Moscow, Russia \\ ${ }^{3}$ Center for Neurobiological Diagnosis of Genetic Psychiatric Disorders, \\ Moscow State University of Psychology and Education, Moscow, Russia
}

Received 13 November 2011; Accepted 18 December 2011

Academic Editors: M. L. Arbonés, T. Arendt, and C. Czech

A well-recognized theory of Alzheimer's disease (AD) pathogenesis suggests ectopic cell cycle events to mediate neurodegeneration. Vulnerable neurons of the AD brain exhibit biomarkers of cell cycle progression and DNA replication suggesting a reentry into the cell cycle. Chromosome reduplication without proper cell cycle completion and mitotic division probably causes neuronal cell dysfunction and death. However, this theory seems to require some inputs in accordance with the generally recognized amyloid cascade theory as well as to explain causes and consequences of genomic instability (aneuploidy) in the AD brain. We propose that unscheduled and incomplete DNA replication (replication stress) destabilizes (epi)genomic landscape in the brain and leads to DNA replication "catastrophe" causing cell death during the S phase (replicative cell death). DNA replication stress can be a key element of the pathogenetic cascade explaining the interplay between ectopic cell cycle events and genetic instabilities in the AD brain. Abnormal cell cycle reentry and somatic genome variations can be used for updating the cell cycle theory introducing replication stress as a missing link between cell genetics and neurobiology of AD.

KEYWORDS: Alzheimer's disease, aneuploidy, cell cycle, DNA replication, replication stress 


\section{INTRODUCTION}

Alzheimer's disease (AD) is a serious public health problem representing one of the commonest causes of dementia in the elderly. Affecting tens of millions of people worldwide, AD is predicted to increase almost twice the frequency during the next 30 years [1]. Although genetic determinants of early-onset AD as well as several genomic loci and risk-enhancing genetic polymorphisms (CNVs or SNPs) of sporadic or late-onset $\mathrm{AD}$ have been determined, the overwhelming majority of cases $(>95 \%)$ does not demonstrate reproducible associations with a specific genetic defect, and the etiology of $\mathrm{AD}$ in these cases remains largely unknown $[2,3]$. Nonetheless, a line of evidences suggests that at least two major molecular (cellular) pathways are implicated in pathophysiology of $\mathrm{AD}$. The first one considers the deposition of amyloid $\beta$-peptide (A $\beta)$ as driving $\mathrm{AD}$ pathogenesis, whereas the second one elucidates abnormal cell cycle events as a cause of a discontinuous change of neuronal cell state/phenotype. However, regardless of the implication in AD neuropathology, these pathways are usually considered apart and the complex interplay between these two cycles (amyloid deposition cycle and abortive cell cycle) remains elusive [4-8].

The commonly accepted cell cycle theory of AD has been first proposed more than 15 years ago and has been repeatedly reviewed previously [7-10]. Since that time, numerous inputs in this theory have been made. More precisely, DNA replication in the AD brain was suggested as a consequence of reentry into the neuronal cell cycle preceding cell death (neurodegeneration) [11]; chromosome instability mediated by aneuploidy of chromosome 21 (probably resulting from cell cycle errors) was found to affect the AD brain [12]; selective neuronal death of aneuploid neurons was proposed to be a cause of progressive AD neurodegeneration [13]; numerous markers (protein biomarkers) of neuronal cell cycle were discovered in the AD brain $[14,15]$. Finally, evidences for neuronal cell cycle events have led to hypothesize that failures of cell cycle regulation can be a root cause of $\mathrm{AD}$ neurodegeneration $[8,16]$. Indeed, all these phenomena in neuronal cells have not been incorporated into an integrated pathway that is able to determine the meaning of "cell-cycle-related" processes in the diseased brain. In an attempt to fill this gap, we propose a hypothesis that is based on commonly accepted cell cycle theory of AD with special attention to the main event of the cell cycle period of DNA synthesis or S phase. Taking into account the available data, it was possible to extend this theory by suggesting inefficient DNA replication that causes DNA replication stress and replication-stress-induced DNA damage in neuronal cells as a missing element of AD pathogenetic cascade. DNA replication stress hypothesis of AD is able to provide a possible link between somatic genome instability, "A $\beta$ deposition," and "cell cycle" pathways. Additionally, empirical testing of the hypothesis is likely to help understanding the genomic mechanisms of neurodegenerative processes in the AD brain.

\section{THEORETICAL BACKGROUND}

The main principles of cycle theory of $\mathrm{AD}$ can be postulated as follows: (i) quiescent postmitotic neurons (previously entered stationary G0 cell cycle phase) unexpectedly reenter into the cell cycle (G1 phase); (ii) incompletely identified mitogenic stimuli probably induce the reentry of neurons into the cell cycle mediated by expression of cell cycle checkpoints (cell cycle regulatory proteins); (iii) diploid neurons (chromosome number: 2N, DNA content: $2 \mathrm{C}$ ) replicate DNA during the $\mathrm{S}$ phase, and duplicated chromosomes possess two unseparated sister chromatids (chromosome number: 2N, DNA content: 4C) in contrast to "true" tetraploidy (chromosome number: 4N, DNA content: 4C); (iv) neurons completely replicate genomic DNA, a transition to the G2 phase occurs, but M phase is not achieved and neurons are arrested at the G2/M transition; (v) genomic content of a neuron passing from S to G2 phase is likely to change from diploid to "tetraploid" or 4C; (vi) changes of genomic landscape and genomic instabilities arising from abortive cell cycle seem to accumulate during ontogeny; (vii) since mitotic phase is not achieved, "G2-arrested neurons" with duplicated genomic content are likely to experience cell death [7, 9-11, 14-19]. A simplified schematic representation of the cycle theory of AD is given in Figure 1.

A critical point of this theory is referred to DNA replication, which is the key event in S phase and is essential for completing cell division. It seems that replicative DNA synthesis in terminally differentiated 


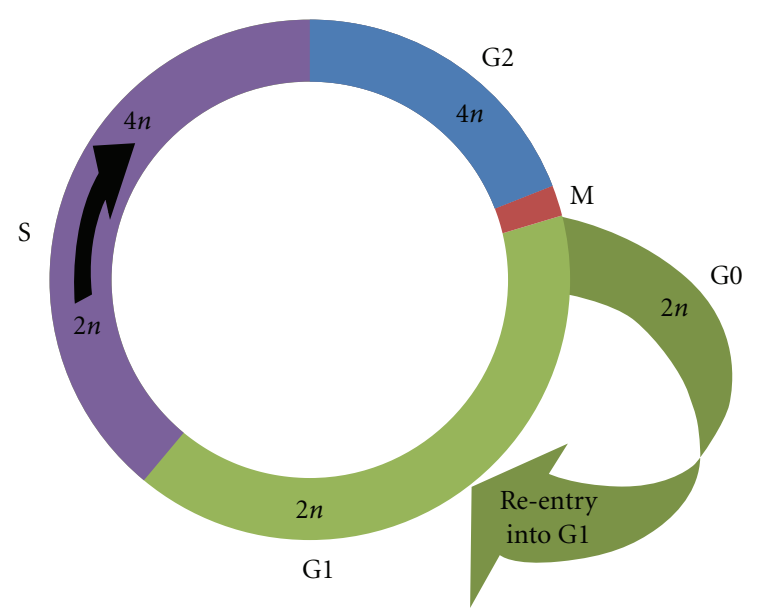

FIGURE 1: Simplified schematic presentation of the cell cycle theory of AD. Quiescent neuronal cells (G0 phase) demonstrate the cell cycle reactivation by either endogenous or environmental mitogenic stimuli followed by reentry into the $\mathrm{G} 1$ phase. The G0/G1 phase transition is critical for a postmitotic neuron and potentially causes neuronal cell death. During $\mathrm{G} 1$ phase, diploid neurons (chromosomal complement: 2N; number of chromosomes: 46; DNA content: 2C) demonstrate G1-specific cell cycle markers (cyclin $\mathrm{D}$ and CDK4/6 complex, cyclin E and CDK2 complex) which are involved in the regulation of $\mathrm{G} 1$ phase progression. Cells successfully passing $G 1$ enter the $S$ phase (phase of DNA replication). During the $S$ phase, CDK2/cyclin E should be silenced to repress additional round of replication of genomic DNA. Protein markers of the $S$ phase are A-type cyclins (cyclin A/CDK2 complex). This complex is essential for proper completion of $S$ phase and transition from $S$ to $G 2$ phase. DNA content of cells during $S$ phase changes from $2 \mathrm{C}$ to $4 \mathrm{C}$ (chromosome number is still $2 \mathrm{~N}$, but DNA content after replication is tetraploid). During G2 phase, cyclin A is degraded and cyclin B/CDC2 complex (protein biomarker of late S/early G2 phases) is formed. Cyclin B/CDC2 complex is essential for triggering mitosis. Neuronal cells in $\mathrm{G} 2$ phase demonstrate tetraploid (4N) DNA content or, more precisely, possess a nucleus with 46 replicated chromosomes. Chromosomal complement (genomic content) of cells in $\mathrm{G} 2$ consists of one set of 46 duplicated chromosomes (DNA content: $4 \mathrm{~N}$ or $4 \mathrm{C}$ : diploid nucleus with replicated chromosomes, for more details see [20]), each having two chromatids- "mitotic" tetraploidy. It is to note that true constitutional polyploidy is a term used to describe cell containing more than two homologous sets of chromosomes (4N or 92 chromosomes, DNA content: $4 \mathrm{C}$ ). We suggest that postmitotic neurons are able to replicate DNA but are not able to make a G2/M transition and divide into two daughter cells.

neurons is a conditionally pathogenic response to endogenous or exogenous stimuli [18]. If DNA replication is processed completely, "affected neurons" would exhibit corresponding cell cycle protein biomarkers specific for G2 (i.e., Cyclin B, CDC2/CDK1) and "genetic biomarkers" such as a doubling of DNA content (4C) and chromosome duplication without separation of sister chromatids (chromosome number: $2 \mathrm{~N}$ and "tetraploid" DNA content: 4C) [11, 14, 15, 18]. Meanwhile, incomplete DNA replication, being highly probable in postmitotic cells, causes replication forks to progress slowly or to stall resulting in $\mathrm{S}$ phase arrest or replication stress. The latter leads to DNA damage or improper repair and, thereby, to accumulation of genomic instabilities [21, 22]. Abnormal DNA replication in postmitotic neurons is likely to be a source of intercellular variations of genomic content $(2 \mathrm{C} \rightarrow 4 \mathrm{C})$ in the $\mathrm{AD}$ brain $[11,12,23,24]$. These are almost the same as those produced by replication stress in other human tissues [25, 26]. In addition, brain-specific aneuploidy, which is found to be implicated in $\mathrm{AD}$ pathogenesis $[12,13,19,23,27,28]$, can result from polyploidy [20, 29, 30], occurring before neuronal differentiation. Finally, genomic instabilities, in parts resulting from replication stress, have been repeatedly associated with neurodegeneration in aging-related diseases [31-34].

Another feature of $\mathrm{AD}$ neurodegeneration that is closely related to numerical chromosome abnormalities (aneuploidy) is referred to neuropathological parallels between AD and Down syndrome 
(DS or trisomy of chromosome 21). In both sporadic and familial ADs, increased chromosome aneuploidy involving different chromosomes, particularly trisomy 21, in neurons and other cells is observed. It is noteworthy, that Down syndrome patients with trisomy 21 develop early onset AD pathology [27, 35]. It has been hypothesized that $\mathrm{AD}$ can be associated with mosaic tissue-specific aneuploidy of chromosome 21, being, in fact, a kind of mosaic DS [36]. This hypothesis has remained largely unproven because of controversial results obtained by different studies for several decades [19, 33, 37-42], until it was experimentally proved by the direct evaluation of chromosome number 21 and structure in the AD brain [12]. Since somatic mosaicism mediated by mitotic aneuploidy is likely to be produced by abnormal mitotic/cell cycle checkpoint [20, 29, 43, 44], an opportunity to link chromosome 21 aneuploidy and cell cycle events in the AD brain seems to exist. To define a relationship between these two peculiarities of genome behavior in the AD brain, origins of aneuploidy in the diseased brain, which remain incompletely established, have to be considered. In an attempt to do so, one can notice a paradox: accumulation of genomic instabilities mediated by cell cycle errors suggests the completion of mitotic division, whereas postmitotic neurons are unlikely to process through the entire cell cycle. In this context, chromosome instability/aneuploidy can only be generated before neuronal differentiation or, in other words, during prenatal or early postnatal brain development (more than 50 years before the average age of AD manifestation). Additionally, human adult neurogenesis is not efficient enough to produce such amount of cells affected by genomic instability, even though neurogenesis is suggested to be enhanced in neurodegenerative diseases [45]. Finally, the amount of neuronal cells exhibiting abnormal "cell cycle phenotypes" is significantly lower $[11,16,18]$ than those affected by genome/chromosome instability $[12,13]$. To solve the paradox, a hypothesis proposing widespread genetic instability to accumulate during ontogenetic periods of extensive neurogenesis was recently proposed. According to this hypothesis, chromosome 21 aneuploidy persists throughout ontogeny until AD manifestation under natural selection (for more details see [19, 33, 41]). The latter is in agreement with observations of genomic instabilities in aging-related diseases and with "intrauterine programming" theory (DNA damage accumulation is supposed to be restricted to the embryonic period producing aging phenotypes during later ontogeny) $[33,41,46]$. The support of these assumptions can also be acquired from data on genomic content of cells populating the developing human brain, about one-third of which are aneuploid being extensively affected by chromosome instability [47-49]. However, all these observations appear to be parts of the AD pathogenetic puzzle missing an important element that is able to link genomic instabilities in the diseased brain, cell cycle events in postmitotic neurons, and AD neuropathology. Finally, the possible link between $\mathrm{DS}$ and $\mathrm{AD}$ needs further experimental testing to establish causal relationship between brain pathology and nonmosaic trisomy 21 due to meiotic chromosome missegregation (the majority of DS patients) or mitotic mosaic trisomy in rare cases of DS and, probably, AD patients. Somatic mosaicism in mosaic $\mathrm{DS}$ and $\mathrm{AD}$ could be the result of chromosome nondisjunction at the earliest stages of the development. Alternatively, aneuploid neurons in the AD brain are likely to result from aberrant adult neurogenesis and gradual accumulation of aneuploid neurons, that is, due to mitogenic activity of plaque forming the proteins [27]. Therefore, accumulation of $\mathrm{A} \beta$-peptide in the brain can be suggested to associate with overproduction of aneuploid neurons during ontogeny and brain aging $\mathrm{AD}$ [27].

\section{HYPOTHESIS: DNA REPLICATION STRESS-A MISSING ELEMENT OF AD PATHOGENETIC CASCADE}

Replication stress or inefficient DNA replication usually originates from alterations to DNA synthesis during $\mathrm{S}$ phase, abnormal expression of replication factors and DNA-binding proteins, and specificity of a number of DNA structures that are difficult to process by replicative machinery [21, 50]. Among the most likely consequences of replication stress, if unrecovered by the genome maintenance apparatus, are failures of DNA repair within specific genomic loci triggering genomic/chromosome instability [50, 51]. Generated during the $\mathrm{S}$ phase, alterations to chromosomes are maintained throughout the rest of cell cycle and are 
observed in daughter cells [50]. These can manifest as polyploidy and aneuploidy generated during the next cellular divisions of polyploid cells $[25,26,50]$. In the light of $\mathrm{AD}$, it is to note that replication stress and its consequences are more frequent in aging tissues and are probably caused by exhaustion of DNA replication and repair machineries [52]. Once produced by replication stress, genomic instabilities persist through subsequent cell divisions as exemplified by studies of cancers [25, 26]. However, high rates of genomic instabilities (produced by replication stress and aneuploidy) can trigger tissue degeneration due to aneuploid cell death in as much as such damage to cellular genomes is incompatible with normal cell homeostasis [53-56]. Similar pathway, in our opinion, seems to be applicable to AD.

Two major molecular and cellular pathways both of which can be produced by genetic defects (i.e., PS1 and APP gene mutations, trisomy of chromosome 21) and/or environmental factors-amyloid deposition and abortive cell cycle — are likely to lead to Alzheimer's type dementia $[2-8,36]$. Interestingly, mutations of $\mathrm{AD}$ genes (presenilins and $A P P$ ) not only lead to increasing the production of $\mathrm{A} \beta$ and contribute to deposition of amyloid but also induce chromosome missegregation (generation of aneuploidy) and are involved in activation of neuronal mitotic/cell cycle checkpoint in the AD brain [2-5, 27, 28, 57, 58]. The activation is likely to underlie the abortive cell cycle in $\mathrm{AD}[7,8,11,14,16,59]$ and to be a likely process enhancing the adult neurogenesis $[45,60]$ producing cells, genomic content of which is actually unknown. Abortive cell cycle in neuronal cells (cell cycle re-entry) is likely to begin at G0-G1 transition $[11,14,16,17]$. Then, these cells have the potential to enter the $S$ phase, to complete DNA replication, and to enter the G2 phase; however, the cell cycle is arrested at the G2-M transition [11, 16-18]. The main outcome of this course of events is the presence of neurons with duplicated chromosomes or "tetraploidy" (tetraploid DNA content), which should be arrested in G2 phase (chromosomes with unseparated sister chromatids, chromosome content: $2 \mathrm{~N}$ ) $[11,15,16]$. However, evaluations of tetraploidy in the AD brain did not demonstrate this chromosome complement in neuronal cells of brain areas affected by neurodegeneration $[12,19,61]$. Nevertheless, cell cycle protein markers specific for both $\mathrm{S}$ and $\mathrm{G} 2$ phases are repeatedly found in the AD brain [7-11, 14-18, 24, 62]. This suggests that cells may enter the $\mathrm{S}$ phase, but the G2 phase is not really achieved because of the lack of tetraploid cells. The discrepancy between data on genomic content (the presence of diploid and hyper-/hypoploid (aneuploid) cells instead of tetraploidy) and cellular biochemical profiling (immunoreactivity for G2 phase biomarkers) is paradoxical. A likely explanation of this paradox could be the "imitation" of cell cycle events (i.e., G2 phase) in vulnerable diploid neurons: cells exhibit biomarkers of G2 phase without complete replication of genomic DNA. Additionally, it is reasonable to propose that initiation and progression of unscheduled DNA synthesis may lead to accumulation of genomic instabilities, abortive DNA replication, and arrest of neuronal cells in S phase. DNA replication stress affects cellular homeostasis and can cause "DNA replication catastrophe" followed by cell death in abortive $\mathrm{S}$ phase (replicative cell death).

Both replication stress and chromosome missegregation are among abnormal cell cycle events usually triggering the accumulation of genomic instabilities [20, 22, 25-30, 41-44, 53-56, 59]. Replication stress, indeed, might result from genomic instability due to replication fork staling of disrupted DNA structures (chromosomal/DNA breaks) [22] as well as can initiate abnormal intracellular processes leading to cell death during the $\mathrm{S}$ phase $[11,16,23]$. Another unresolved paradox is related to the abundance of chromosome 21 aneuploidy in the AD brain [12], whereas cell cycle errors and replication stress usually produce multiple types of genome/chromosome instability (i.e., tetraploidy or unreleased DNA/chromosome breaks) $[15,20,22,25,26,29-31]$. In other words, the source of aneuploidy in the human adult brain remains intriguingly unknown [19, 33, 41, 59]. Currently, origins of mosaic aneuploidy are assumed to result essentially from chromosomal nondisjunction during mitosis at early stages of ontogeny (prenatal development), and chromosome specificity of aneuploidy is likely to be achieved under natural selection within cellular populations [41, 48, 63-65]. Thus, we have to admit that cells with chromosome 21 trisomy possess some advantages in terms of cellular physiology [33, 41, 64, 65]. Together, one can conclude that, despite of the accumulation of genomic instabilities in the diseased brain, 
those cells affected by chromosome 21 aneuploidy are potentially more viable than either tetraploid or non-chromosome-21-aneuploid cells. In addition, chromosome instability manifested as aneuploidy is an integral part of the developing human brain probably involved in generation of neuronal variability and complexity $[44,47-49,54,66]$, experiencing, however, a significant decrease ( $~ 3$ times) throughout further ontogenetic stages [12, 23, 47, 49, 59]. Therefore, selective pressure against aneuploid neurons is likely to exist in the unaffected human brain but seems to be altered in the brain of individuals affected by neurodegenerative diseases [18, 32-34, 41, 59, 64]. Moreover, other types of genomic instabilities arising from abnormal DNA repair (including those produced by replication stress) are likely to be generated and to accumulate during prenatal development regardless of the manifestation at later ontogeny [33, 46]. Alternatively, the involvement of chromosome 21 aneuploidy in $\mathrm{AD}$ pathogenesis is supported by the fact that $A P P$ - a key element of the AD pathogenetic cascade-is located on chromosome 21 [2-6]. Furthermore, several indirect evidences for a contribution of aneuploidy of chromosome 21 and genomic instability to AD neurodegeneration such as shared susceptibility to aneuploidy and AD in mothers of DS children as well as markers of genome/chromosome instability (increased frequency of micronuclei) and oxidative stress associated with genomic instabilities in AD individuals have been previously provided [6769]. Since aneuploidy causes additional copies of $A P P$ (as exemplified by studies of DS), an opportunity to link genomic instability manifested as chromosome 21 aneuploidy with $\mathrm{A} \beta$ aggregation, deposition, and accumulation does exist $[33,35,36,39]$. On the other hand, it has been recently shown that $A P P$ and $\mathrm{A} \beta$ are involved in mitotic/cell cycle abnormalities in neuronal cells associated with AD [27, 28]. Therefore, abnormal cell cycle events can be linked to amyloid deposition cycle through one key element of AD pathogenetic cascade-replication stress. The graphical overview of the replication stress hypothesis of AD is presented by Figure 2 .

Although the role of DNA replication and numerical chromosome abnormalities in cellular pathology associated with Alzheimer's type dementia has been proposed [11,23], variable genomic landscape of neuronal cells experiencing cell death and cellular phenotype suggests a more complex interplay between cell cycle events, genetic instabilities, and neurodegeneration [12, 13, 23, 27, 61]. The present hypothesis is an attempt to provide a more general explanation of how all these data can be processed in order to give an integrated view of interplay between cell cycle events, amyloid deposition (increased A $\beta 42$ ), and genomic instabilities affecting the AD brain. Since DNA replication has been consistently considered a critical event in abnormal neuronal cell cycle [11,23], we have proposed to make a closer look at this phenomenon and to incorporate it into the unifying pathway for molecular and cellular mechanisms of AD.

\section{IMPLICATIONS}

The possibility to consider abnormal cell cycle events in the AD brain as therapeutic targets has been repeatedly proposed $[7,8,19,61,70]$. However, due to the lack of integrated view of cell cycle $\mathrm{AD}$ pathogenetic cascade, it was difficult to be more specific for selection of molecular interventions. Establishing the missing links in pathogenetic pathways seems to provide a closer, molecular-oriented look at possible therapies of this common and devastative neurodegenerative disease.

Testing DNA replication stress hypothesis can make a contribution to our understanding of the disease course and provide for identifying the sequence of abnormal events that generate pathologic cellular and organism phenotype. The latter is important for biomarker identification and development of preclinical molecular tests (i.e., analysis of chromosomal aneuploidy and genome instabilities, abnormal replicative activity of neural cells, etc.) before the manifestation of disease and, more importantly, identification of endogenous and environmental factors that promote abnormal cell cycle reentry and replication stress, which can be avoided by individuals at risk. Finally, the knowledge about the way abnormal cell cycle events and genomic/chromosome instabilities occur in the $\mathrm{AD}$ brain would allow disease prevention that is undoubtedly easier than a development of molecular therapies to treat the disease after clinical manifestation. 


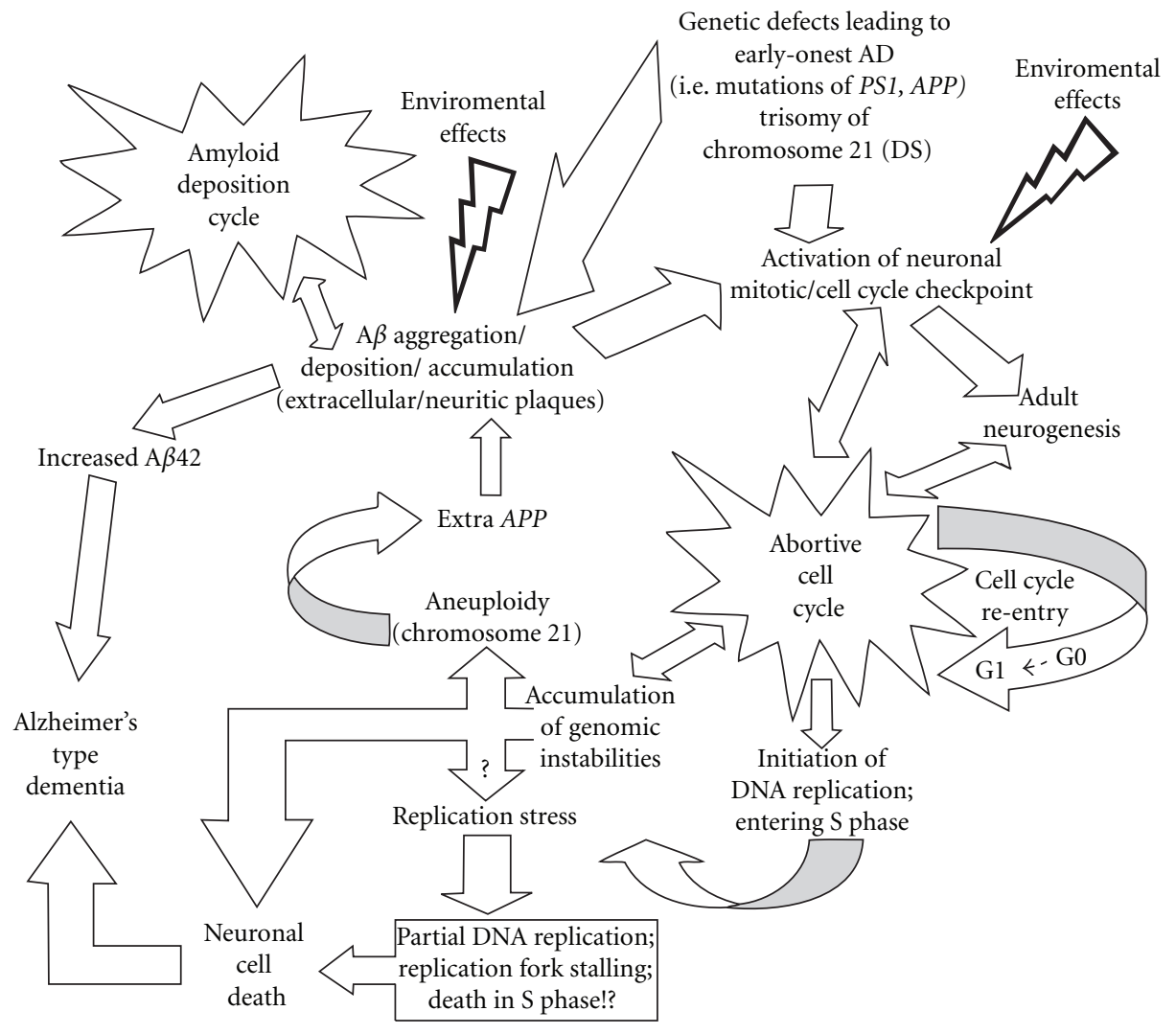

FIGURE 2: The DNA replication stress hypothesis of AD. Interplay between essential elements of the ADtype dementia pathogenetic cascade is proposed. The genetic influences (PSEN or APP mutations, trisomy 21, APOE4 genotype), metabolic changes, and environmental factors affecting neuronal homeostasis in the aging brain lead to activation of neuronal proliferation. Mitogens, which do exist in the human brain (neuronal cells), induce additional stimuli of extensive adult neurogenesis in the hippocampus. In the AD brain, such events would lead to increased hippocampal neurogenesis. A side effect could be that these mitogenic stimuli activate cell cycle reentry in postmitotic neurons. The latter is a pathological activation of neuronal cell cycle, including reentry into $\mathrm{G} 1$ and $\mathrm{S}$ phases and initiation of DNA replication. Neurons showing protein markers of G2/M phase, probably, contain chromosome set of 23 duplicated chromosome pairs with unseparated chromatids (DNA content-4C; chromosome complement: $2 \mathrm{~N}$ ) and become tetraploid in a sense of DNA content (4C). According to the commonly accepted theory of neuronal cell cycle reentry and death, some neuronal populations complete the DNA synthesis but are arrested during the G2/M transition. Therefore, neuronal death occurs in G2 phase. Alternatively, one can propose that a large proportion of activated postmitotic neurons in the AD brain are unable to pass properly the $S$ phase. This would lead to accumulation of genomic and chromosomal instabilities throughout ontogeny (DNA breaks, aneuploidy). In addition, replication-induced DNA damages would lead to fork stalling, incomplete or inefficient DNA replication, together designated as replication stress. Replication stress may be considered the leading cause of neuronal cell death due to processing into $S$ phase or accumulation of genetic instabilities, which together constitute an important element of the $A D$ pathogenetic cascade. According to the present hypothesis, the possibility to link the two main pathways of $A D$ arises from the introduction of accumulation of genomic instabilities associated with DNA replication stress, which is able to produce as neuronal cell death (replicative cell death) as chromosomal aneuploidy due to natural selection in neural cell populations probably causing extra APP in the diseased brain. 


\section{CONCLUSION}

Solving the paradox of AD cell cycle theory arising from discrepancies between data on cell cycle biomarkers (i.e., G2-markers) and genomic content of neuronal cells leads to a speculation that neurons entered into $\mathrm{S}$ phase do not proceed further through the cell cycle. This suggests neuronal cell dysfunction and death to occur during the $\mathrm{S}$ phase (death in $\mathrm{S}$ phase) and to result from replication stress. In others words, unscheduled and unrealized DNA synthesis in vulnerable neurons should lead to DNA replication catastrophe and neuronal death due to lethal errors in replication (replicative cell death). The latter is likely to be partially caused by the impossibility of epigenetic reorganization of the nuclear genome for proper chromosome duplication. In this context, G2 phase biomarkers are likely to be a sign of cell cycle "imitation" or other intracellular phenomena accompanied by expression of cell-cycle proteins but not directly related to cell cycle events (i.e., DNA reparation, DNA damage response, and initiation of programmed cell death). DNA replication stress is a probable trigger of genome instability in the AD brain, which links abnormal cell cycle events, chromosomal aneuploidy, and $\mathrm{A} \beta$ production and deposition. Testing of the "DNA replication stress" or "DNA replicative death" hypothesis of AD would help to expand our views of how neural cell cycle dysregulation is involved in AD pathogenesis. Furthermore, it can give insights into current knowledge about the role of adult neurogenesis in the normal brain development and in the development of brain disease and the way how genome (epigenome) stability is maintained in neuronal cells through the lifespan.

Since abnormal cell cycle regulation has been repeatedly associated with a broad spectrum of neurodegenerative diseases (AD, Parkinson's disease, amyotrophic lateral sclerosis, ataxia-telangiectasia, and Niemann-Pick type $\mathrm{C}$ disease) $[16,18,32,34,54,59]$, a hypothesis proposing replication stress to be a critical event seems to be applicable for neurodegeneration, as a whole. To test the hypothesis, additional molecular cytogenetic and cytogenomic studies of DNA replication pattern and somatic genome instability in the AD brain are needed. These studies dedicated to identifying the role of genome instability and DNA replication in cell-cycle-mediated neuropathology would lead to establishing the etiology and pathogenesis of sporadic $\mathrm{AD}$, which encompasses the majority of $\mathrm{AD}$-type dementia. If confirmed, the present hypothesis, postulating the existence of a cascade of events including "DNA replication stress," "DNA replication catastrophe" followed by "replicative cell death," would provide for a molecular-oriented definition of neurodegeneration pathways and, as a result, would open new opportunities for molecular interventions in related devastative neurological and psychiatric disorders.

\section{ACKNOWLEDGMENT}

The paper is dedicated to Ilia V. Soloviev. The authors are supported by DLR/BMBF Grants (RUS 09/006 and RUS 11/002).

\section{REFERENCES}

[1] C. Reitz, C. Brayne, and R. Mayeux, "Epidemiology of Alzheimer disease," Nature Reviews Neurology, vol. 7, no. 3, pp. 137-152, 2011.

[2] R. E. Tanzi and L. Bertram, "Twenty years of the Alzheimer's disease amyloid hypothesis: a genetic perspective," Cell, vol. 120, no. 4, pp. 545-555, 2005.

[3] L. Bertram, C. M. Lill, and R. E. Tanzi, "The genetics of alzheimer disease: back to the future," Neuron, vol. 68, no. 2, pp. 270-281, 2010.

[4] J. Hardy and D. J. Selkoe, “The amyloid hypothesis of Alzheimer's disease: progress and problems on the road to therapeutics," Science, vol. 297, no. 5580, pp. 353-356, 2002.

[5] M. Goedert and M. G. Spillantini, “A century of Alzheimer's disease," Science, vol. 314, no. 5800, pp. 777-781, 2006. 
[6] R. H. Swerdlow, "Pathogenesis of Alzheimer's disease," Clinical Interventions in Aging, vol. 2, no. 3, pp. 347359, 2007.

[7] J. Woods, M. Snape, and M. A. Smith, "The cell cycle hypothesis of Alzheimer's disease: suggestions for drug development," Biochimica et Biophysica Acta, vol. 1772, no. 4, pp. 503-508, 2007.

[8] K. Herrup, "Reimagining Alzheimer's disease - An age-based hypothesis," Journal of Neuroscience, vol. 30, no. 50, pp. 16755-16762, 2010.

[9] T. Arendt, L. Rödel, U. Gärtner, and M. Holzer, "Expression of the cyclin-dependent kinase inhibitor p16 in Alzheimer's disease," NeuroReport, vol. 7, no. 18, pp. 3047-3049, 1996.

[10] I. Vincent, M. Rosado, and P. Davies, "Mitotic mechanisms in Alzheimer's disease?" Journal of Cell Biology, vol. 132, no. 3, pp. 413-425, 1996.

[11] Y. Yang, D. S. Geldmacher, and K. Herrup, "DNA replication precedes neuronal cell death in Alzheimer's disease," Journal of Neuroscience, vol. 21, no. 8, pp. 2661-2668, 2001.

[12] I. Y. Iourov, S. G. Vorsanova, T. Liehr, and Y. B. Yurov, "Aneuploidy in the normal, Alzheimer's disease and ataxia-telangiectasia brain: differential expression and pathological meaning," Neurobiology of Disease, vol. 34, no. 2, pp. 212-220, 2009.

[13] T. Arendt, M. K. Brückner, B. Mosch, and A. Lösche, “Selective cell death of hyperploid neurons in Alzheimer's disease," American Journal of Pathology, vol. 177, no. 1, pp. 15-20, 2010.

[14] A. Currais, T. Hortobágyi, and S. Soriano, "The neuronal cell cycle as a mechanism of pathogenesis in Alzheimer's disease," Aging, vol. 1, no. 4, pp. 363-371, 2009.

[15] J. M. Frade and N. López-Sánchez, "A novel hypothesis for Alzheimer's disease based on neuronal tetraploidy induced by p75NTR," Cell Cycle, vol. 9, no. 10, pp. 1934-1941, 2010.

[16] K. Herrup and Y. Yang, "Cell cycle regulation in the postmitotic neuron: oxymoron or new biology?" Nature Reviews Neuroscience, vol. 8, no. 5, pp. 368-378, 2007.

[17] A. Copani, F. Caraci, J. J. M. Hoozemans, M. Calafiore, M. Angela Sortino, and F. Nicoletti, "The nature of the cell cycle in neurons: focus on a "non-canonical" pathway of DNA replication causally related to death," Biochimica et Biophysica Acta, vol. 1772, no. 4, pp. 409-412, 2007.

[18] W. Wang, B. Bu, M. Xie, M. Zhang, Z. Yu, and D. Tao, "Neural cell cycle dysregulation and central nervous system diseases," Progress in Neurobiology, vol. 89, no. 1, pp. 1-17, 2009.

[19] I. Y. Iourov, S. G. Vorsanova, and Y. B. Yurov, "Genomic landscape of the Alzheimer's disease brain: chromosome instability—aneuploidy, but not tetraploidy—mediates neurodegeneration," Neurodegenerative Diseases, vol. 8, no. 1-2, pp. 35-37, 2010.

[20] Z. Storchova and D. Pellman, "From polyploidy to aneuploidy, genome instability and cancer," Nature Reviews Molecular Cell Biology, vol. 5, no. 1, pp. 45-54, 2004.

[21] W. C. Burhans and M. Weinberger, "DNA replication stress, genome instability and aging," Nucleic Acids Research, vol. 35, no. 22, pp. 7545-7556, 2007.

[22] A. C. Bester, M. Roniger, Y. S. Oren et al., "Nucleotide deficiency promotes genomic instability in early stages of cancer development," Cell, vol. 145, no. 3, pp. 435-446, 2011.

[23] B. Mosch, M. Morawski, A. Mittag, D. Lenz, A. Tarnok, and T. Arendt, "Aneuploidy and DNA replication in the normal human brain and Alzheimer's disease," Journal of Neuroscience, vol. 27, no. 26, pp. 6859-6867, 2007.

[24] J. Chen, M. L. Cohen, A. J. Lerner, Y. Yang, and K. Herrup, "DNA damage and cell cycle events implicate cerebellar dentate nucleus neurons as targets of Alzheimer's disease," Molecular Neurodegeneration, vol. 5, p. 60, 2010.

[25] A. Gibaud, N. Vogt, N. S. Hadj-Hamou et al., "Extrachromosomal amplification mechanisms in a glioma with amplified sequences from multiple chromosome loci," Human Molecular Genetics, vol. 19, no. 7, pp. 1276-1285, 2010.

[26] Y. Ichijima, K. I. Yoshioka, Y. Yoshioka et al., "DNA lesions induced by replication stress trigger mitotic aberration and tetraploidy development," PLoS ONE, vol. 5, no. 1, Article ID e8821, 2010.

[27] A. Granic, J. Padmanabhan, M. Norden, and H. Potter, "Alzheimer A $\beta$ peptide induces chromosome missegregation and aneuploidy, including trisomy 21: requirement for tau and APP," Molecular Biology of the Cell, vol. 21, no. 4, pp. 511-520, 2010.

[28] S. I. Borysov, A. Granic, J. Padmanabhan, C. E. Walczak, and H. Potter, "Alzheimer A $\beta$ disrupts the mitotic spindle and directly inhibits mitotic microtubule motors," Cell Cycle, vol. 10, no. 9, pp. 1397-1410, 2011. 
[29] I. Y. Iourov, S. G. Vorsanova, and Y. B. Yurov, "Intercellular genomic (chromosomal) variations resulting in somatic mosaicism: mechanisms and consequences," Current Genomics, vol. 7, no. 7, pp. 435-446, 2006.

[30] Z. Storchova and C. Kuffer, "The consequences of tetraploidy and aneuploidy," Journal of Cell Science, vol. 121, no. 23, pp. 3859-3866, 2008.

[31] A. Nussenzweig, "Causes and consequences of the DNA damage response," Cell Cycle, vol. 6, no. 19, pp. 23392340, 2007.

[32] I. Y. Iourov, S. G. Vorsanova, T. Liehr, A. D. Kolotii, and Y. B. Yurov, "Increased chromosome instability dramatically disrupts neural genome integrity and mediates cerebellar degeneration in the ataxia-telangiectasia brain," Human Molecular Genetics, vol. 18, no. 14, pp. 2656-2669, 2009.

[33] Y. B. Yurov, S. G. Vorsanova, and I. Y. Iourov, "GIN'n'CIN hypothesis of brain aging: deciphering the role of somatic genetic instabilities and neural aneuploidy during ontogeny," Molecular Cytogenetics, vol. 2, no. 1, article 23, 2009.

[34] D. K. Jeppesen, V. A. Bohr, and T. Stevnsner, "DNA repair deficiency in neurodegeneration," Progress in Neurobiology, vol. 94, no. 2, pp. 166-200, 2011.

[35] H. Potter, "Review and hypothesis: Alzheimer disease and Down syndrome—chromosome 21 nondisjunction may underlie both disorders," American Journal of Human Genetics, vol. 48, no. 6, pp. 1192-1200, 1991.

[36] H. Potter, "Down's syndrome and Alzheimer's disease: two sides of the same coin," Future Neurology, vol. 3, no. 1, pp. 29-37, 2008.

[37] K. E. Buckton, L. J. Whalley, M. Lee, and J. E. Christie, "Chromosome changes in Alzheimer's presenile dementia," Journal of Medical Genetics, vol. 20, no. 1, pp. 46-51, 1983.

[38] J. Hardy, A. Goate, M. Owen, and M. Rossor, "Presenile dementia associated with mosaic trisomy 21 in a patient with a Down syndrome child," Lancet, vol. 2, no. 8665, pp. 743-744, 1989.

[39] L. N. Geller and H. Potter, "Chromosome missegregation and trisomy 21 mosaicism in Alzheimer's disease," Neurobiology of Disease, vol. 6, no. 3, pp. 167-179, 1999.

[40] P. Thomas and M. Fenech, "Chromosome 17 and 21 aneuploidy in buccal cells is increased with ageing and in Alzheimer's disease," Mutagenesis, vol. 23, no. 1, pp. 57-65, 2008.

[41] Y. B. Yurov, S. G. Vorsanova, and I. Y. Iourov, "Ontogenetic variation of the human genome," Current Genomics, vol. 11, no. 6, pp. 420-425, 2010.

[42] B. Spremo-Potparević, L. Živković, B. Plećas-Solarović, and V. P. Bajić, “Chromosome instability in Alzheimer's disease," Archives of Biological Sciences, vol. 63, no. 3, pp. 603-608, 2011.

[43] D. Cimini and F. Degrassi, "Aneuploidy: a matter of bad connections," Trends in Cell Biology, vol. 15, no. 8, pp. 442-451, 2005.

[44] I. Y. Iourov, S. G. Vorsanova, and Y. B. Yurov, "Somatic genome variations in health and disease," Current Genomics, vol. 11, no. 6, pp. 387-396, 2010.

[45] B. Winner, Z. Kohl, and F. H. Gage, "Neurodegenerative disease and adult neurogenesis," European Journal of Neuroscience, vol. 33, no. 6, pp. 1139-1151, 2011.

[46] O. Fernandez-Capetillo, "Intrauterine programming of ageing," EMBO Reports, vol. 11, no. 1, pp. 32-36, 2010.

[47] Y. B. Yurov, I. Y. Iourov, V. V. Monakhov, I. V. Soloviev, V. M. Vostrikov, and S. G. Versanova, "The variation of aneuploidy frequency in the developing and adult human brain revealed by an interphase FISH study," Journal of Histochemistry and Cytochemistry, vol. 53, no. 3, pp. 385-390, 2005.

[48] Y. B. Yurov, I. Y. Iourov, S. G. Vorsanova et al., "Aneuploidy and confined chromosomal mosaicism in the developing human brain," PLoS ONE, vol. 2, no. 6, article e558, 2007.

[49] J. W. Westra, S. E. Peterson, Y. C. Yung, T. Mutoh, S. Barral, and J. Chun, "Aneuploid mosaicism in the developing and adult cerebellar cortex," Journal of Comparative Neurology, vol. 507, no. 6, pp. 1944-1951, 2008.

[50] C. Allen, A. K. Ashley, R. Hromas, and J. A. Nickoloff, "More forks on the road to replication stress recovery," Journal of Molecular Cell Biology, vol. 3, no. 1, pp. 4-12, 2011.

[51] D. Branzei and M. Foiani, "The checkpoint response to replication stress," DNA Repair, vol. 8, no. 9, pp. 10381046, 2009.

[52] M. C. Haigis and B. A. Yankner, “The aging stress response," Molecular Cell, vol. 40, no. 2, pp. 333-344, 2010.

[53] B. A. A. Weaver and D. W. Cleveland, "Decoding the links between mitosis, cancer, and chemotherapy: the mitotic checkpoint, adaptation, and cell death," Cancer Cell, vol. 8, no. 1, pp. 7-12, 2005. 
[54] I. Y. Iourov, S. G. Vorsanova, and Y. B. Yurov, "Chromosomal variation in mammalian neuronal cells: known facts and attractive hypotheses," International Review of Cytology, vol. 249, pp. 143-191, 2006.

[55] M. Dierssen, Y. Herault, and X. Estivill, "Aneuploidy: from a physiological mechanism of variance to Down syndrome," Physiological Reviews, vol. 89, no. 3, pp. 887-920, 2009.

[56] J. M. Sheltzer and A. Amon, "The aneuploidy paradox: costs and benefits of an incorrect karyotype," Trends in Genetics, vol. 27, no. 11, pp. 446-453, 2011.

[57] J. Li, M. Xu, H. Zhou, J. Ma, and H. Potter, "Alzheimer presenilins in the nuclear membrane, interphase kinetochores, and centrosomes suggest a role in chromosome segregation," Cell, vol. 90, no. 5, pp. 917-927, 1997.

[58] D. I. Boeras, A. Granic, J. Padmanabhan, N. C. Crespo, A. M. Rojiani, and H. Potter, "Alzheimer's presenilin 1 causes chromosome missegregation and aneuploidy," Neurobiology of Aging, vol. 29, no. 3, pp. 319-328, 2008.

[59] T. Arendt, B. Mosch, and M. Morawski, "Neuronal aneuploidy in health and disease: a cytomic approach to understand the molecular individuality of neurons," International Journal of Molecular Sciences, vol. 10, no. 4, pp. 1609-1627, 2009.

[60] K. Jin, A. L. Peel, X. O. Mao et al., "Increased hippocampal neurogenesis in Alzheimer's disease," Proceedings of the National Academy of Sciences of the United States of America, vol. 101, no. 1, pp. 343-347, 2004.

[61] J. W. Westra, S. Barral, and J. Chun, "A reevaluation of tetraploidy in the Alzheimer's disease brain," Neurodegenerative Diseases, vol. 6, no. 5-6, pp. 221-229, 2009.

[62] U. Ueberham and T. Arendt, "The expression of cell cycle proteins in neurons and its relevance for Alzheimer's disease," Current Drug Targets, vol. 4, no. 3, pp. 293-306, 2005.

[63] M. A. Hultén, S. D. Patel, M. Westgren et al., "On the paternal origin of trisomy 21 Down syndrome," Molecular Cytogenetics, vol. 3, no. 1, article 4, 2010.

[64] I. Y. Iourov, S. G. Vorsanova, and Y. B. Yurov, "Chromosomal mosaicism goes global,” Molecular Cytogenetics, vol. 1, article 26, 2008.

[65] M. A. Hultén, J. Jonasson, A. Nordgren, and E. Iwarsson, "Germinal and somatic trisomy 21 mosaicism: how common is it, what are the implications for individual carriers and how does it come about?" Current Genomics, vol. 11, no. 6, pp. 409-419, 2010.

[66] A. R. Muotri and F. H. Gage, "Generation of neuronal variability and complexity," Nature, vol. 441, no. 7097, pp. 1087-1093, 2006.

[67] L. Migliore, F. Migheli, and F. Coppedè, "Susceptibility to aneuploidy in young mothers of Down syndrome children," TheScientificWorldJournal, vol. 9, pp. 1052-1060, 2009.

[68] F. Coppedè, "One-carbon metabolism and Alzheimer's disease: focus on epigenetics," Current Genomics, vol. 11, no. 4, pp. 246-260, 2010.

[69] L. Migliore, F. Coppedè, M. Fenech, and P. Thomas, "Association of micronucleus frequency with neurodegenerative diseases," Mutagenesis, vol. 26, no. 1, pp. 85-92, 2011.

[70] P. Taupin, "Neurogenesis, NSCs, pathogenesis and therapies for Alzheimer's disease," Frontiers in Bioscience, vol. 3, pp. 178-190, 2011.

\section{This article should be cited as follows:}

Yuri B. Yurov, Svetlana G. Vorsanova, and Ivan Y. Iourov, "The DNA Replication Stress Hypothesis of Alzheimer's Disease,” TheScientificWorldJOURNAL, vol. 11, pp. 2602-2612, 2011. 


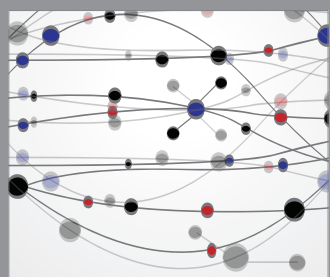

The Scientific World Journal
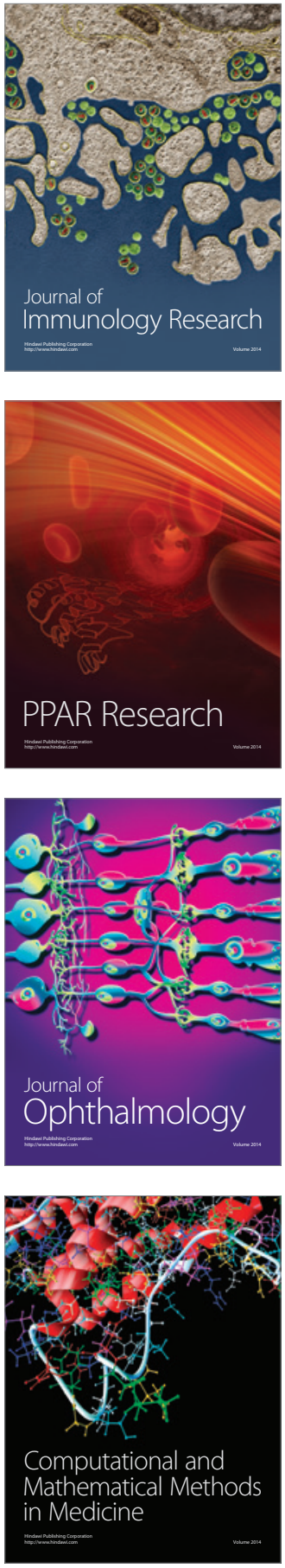

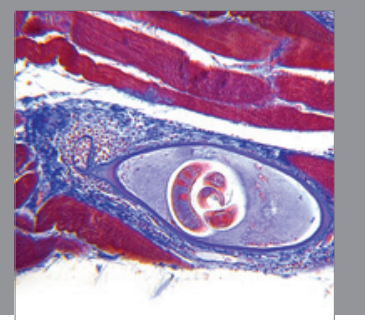

Gastroenterology

Research and Practice
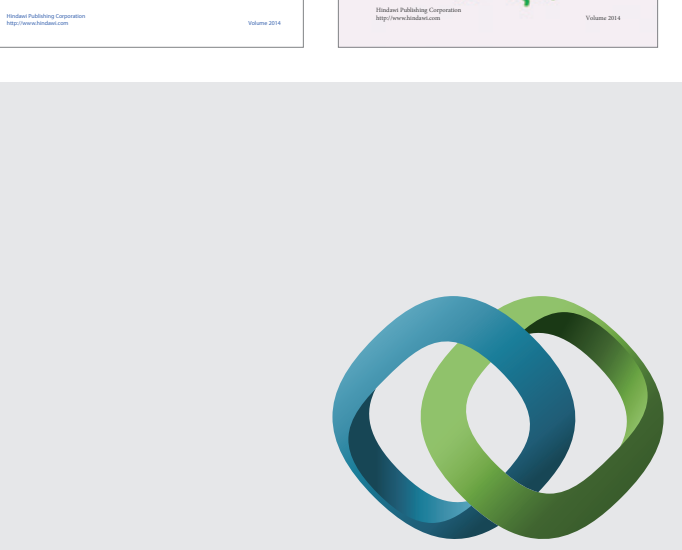

\section{Hindawi}

Submit your manuscripts at

http://www.hindawi.com
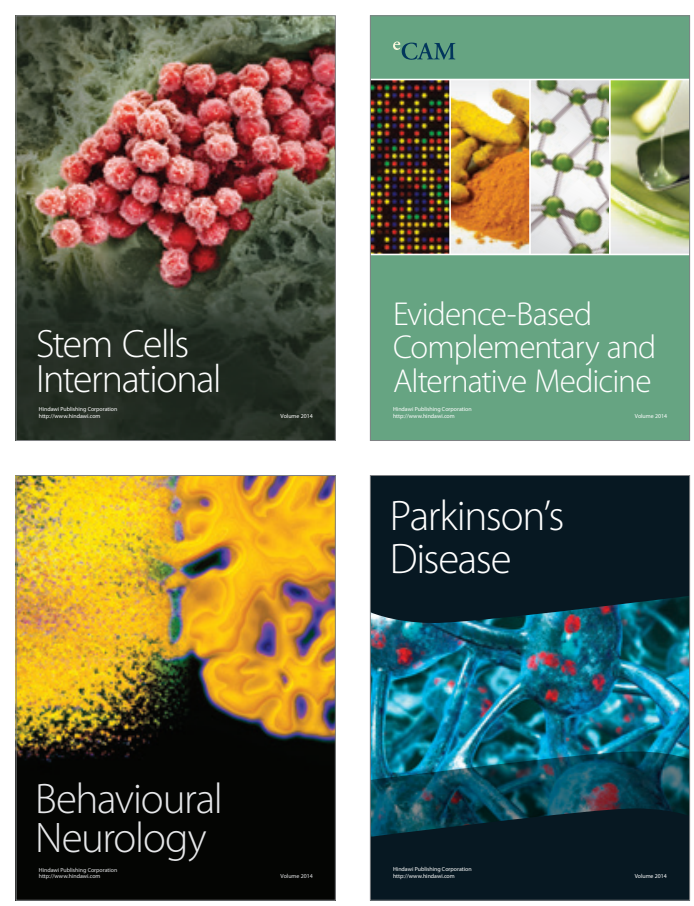

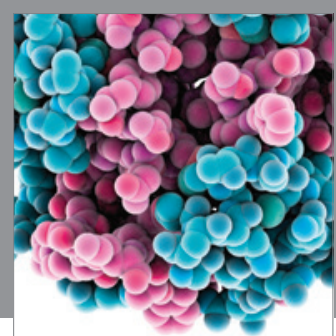

Journal of
Diabetes Research

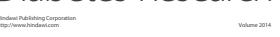

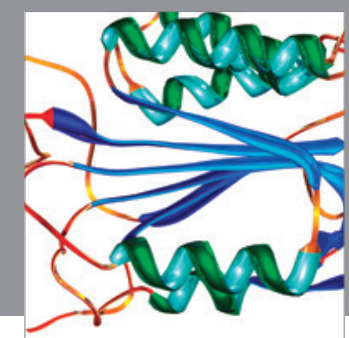

Disease Markers
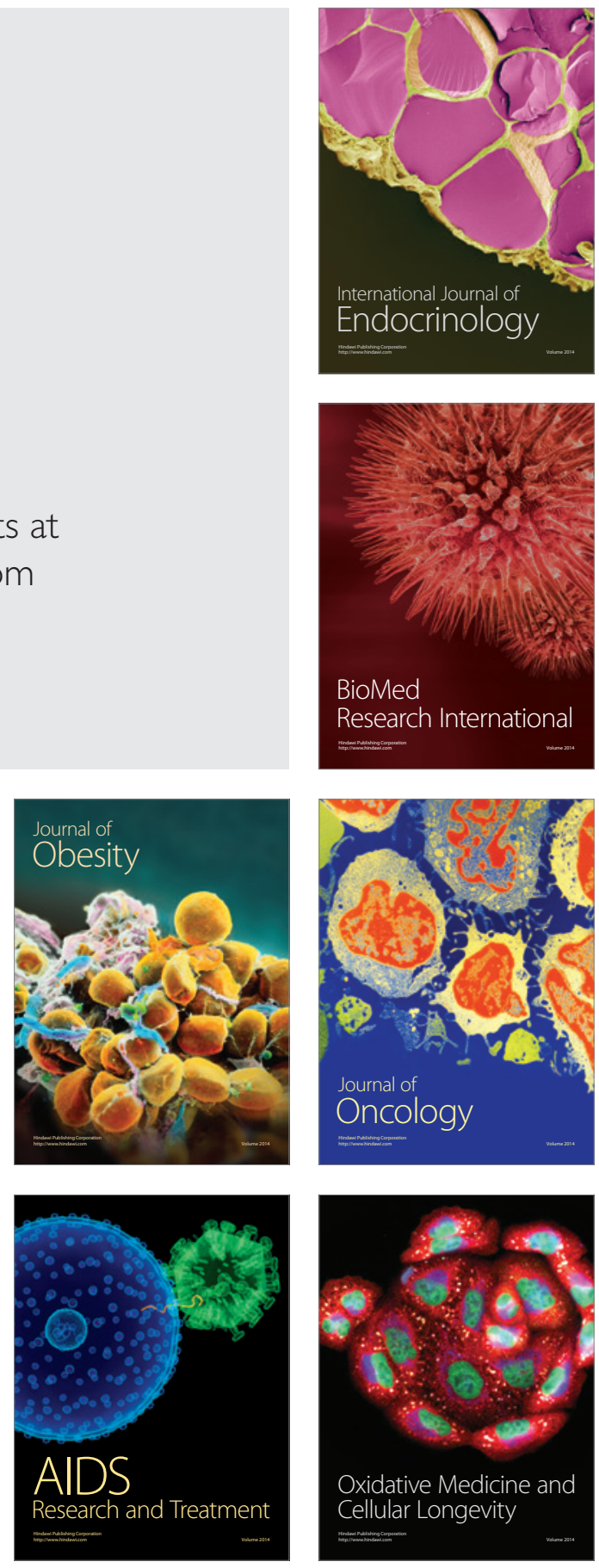